\title{
Problemas relacionados ao uso de álcool e outras drogas entre alunos de uma Escola Municipal de Uberaba - um estudo piloto*
}

\section{Problems related to the use of alcohol and other drugs among students of a Municipal School in Uberaba - a pilot study}

\author{
Andrea Ruzzi Pereira1, Rafaela Caroline Silveira de Castro ${ }^{2}$, \\ Jair Lício Ferreira Santos ${ }^{3}$
}

http://dx.doi.org/10.11606/issn.2238-6149.v27i3p263-270

\begin{abstract}
Ruzzi-Pereira A, Castro RCS, Santos JLF. Problemas relacionados ao uso de álcool e outras drogas entre alunos de uma Escola Municipal de Uberaba - um estudo piloto. Rev Ter Ocup Univ São Paulo. 2016 set.-dez.;27(3):263-70.
\end{abstract}

RESUMO: Contexto: O consumo de álcool e outras drogas por adolescentes têm crescido nos últimos anos, considerado um problema de saúde pública, razão para estudos e envolvimento da sociedade na busca de soluções preventivas. Objetivo: Avaliar o risco de problemas relacionados ao uso de álcool e outras drogas entre estudantes do $6^{\circ}$ ao $9^{\circ}$ ano de uma escola pública do ensino fundamental. Métodos: A amostra foi definida pelo critério de amostragem não probabilístico, com participação de 55 adolescentes. Os dados foram obtidos mediante a aplicação do inventário DUSI - Drug Use Screening Inventory, que é composto por 149 questões divididas em 10 áreas. Resultados: Não há diferença entre sexo na intensidade geral de problemas. As áreas com maior risco de problemas referem-se ao sistema familiar e ao lazer/recreação. Os sujeitos de 10 anos apresentam risco menor de problemas, comparados à média geral do grupo em relação aos sujeitos mais velhos. A análise das médias da densidade mostrou que meninos e meninas apresentam resultados semelhantes na maioria das áreas. Conclusão: Há necessidade de investimentos em lazer/recreação saudáveis e adequados, visto essa ser a área de maior risco para problemas associados ao uso de álcool e outras drogas.

DESCRITORES: Detecção do abuso de substâncias; Fatores de risco; Adolescente.
Ruzzi-Pereira A, Castro RCS, Santos JLF. Problems related to the use of alcohol and other drugs among students of a Municipal School in Uberaba - a pilot study. Rev Ter Ocup Univ São Paulo. 2016 Sept.-Dec.;27(3):263-70.

\begin{abstract}
Context: The consumption of alcohol by adolescents has increased in the last few years, considered a public health issue, reason for studies and society's involvement in the search of preventive solutions. Objective: To evaluate the risks of problems related to the use of alcohol and other drugs among students from $6^{\text {th }}$ to $9^{\text {th }}$ grade of an elementary education public school. Methods: The sample was defined by the non-probability sampling criterion, with participation of 55 adolescents. The data were collected through the application of the Drug Use Screening Inventory - DUSI, which is composed of 149 questions divided into 10 areas. Results: There is no difference between genders in the general intensity of problems. The areas with highest risk of problems refer to familiar system and leisure/recreation. The 10-year-old subjects present the lowest risk of problems, compared to the group's general average in relation to the older subjects. The analysis of the density averages has shown that boys and girls present similar results in most areas. Conclusion: There is need of investment in healthy and appropriate leisure/ recreation, once this is the area of highest risk of problems associated to the use of alcohol and other drugs.
\end{abstract}

KEYWORDS: Substance abuse detection; Risk factors; Adolescent.

\footnotetext{
*Resultado do trabalho de conclusão de curso (TCC) do Curso de Terapia Ocupacional da Universidade Federal do Triângulo Mineiro (UFTM) - Aprovado Pelo Comitê de Ética da UFTM de acordo com o parecer N ${ }^{\circ}$ 2439/2013. Também utilizado como estudo piloto para o trabalho de doutorado que teve como produto a tese intitulada "Prevalência do uso de álcool e outras drogas entre escolares da rede pública de ensino de Uberaba - MG: fatores de risco e de proteção".

1. Universidade Federal do Triângulo Mineiro, Departamento de Terapia Ocupacional. Email: ruzzi.pereira@gmail.com

2. APAE Montes Claros, MG; APAE Janaúba, MG. Email: rafacastro.to@hotmail.com.

3. Hospital das Clínicas da Faculdade de Medicina de Ribeirão Preto da Universidade de São Paulo (HC-FMRP-USP), Departamento de Medicina Social. Email: jalifesa@usp.br

Endereço para correspondência: Universidade Federal do Triângulo Mineiro. Unidade Centro Educacional (CE). Departamento de Terapia Ocupacional. Av. Getúlio Guaritá, 159. $3^{\circ}$ Piso. Uberaba, MG. CEP: 38025-440. E-mail: ruzzi.pereira@gmail.com
} 


\section{INTRODUÇÃO}

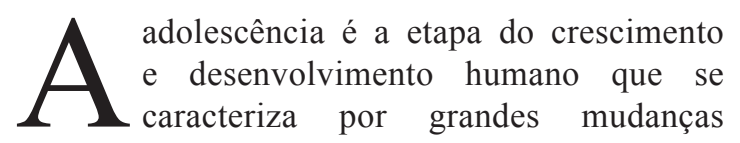
físicas, psíquicas, sociais e emocionais que, de acordo com alguns autores, pode se estender dos 10 aos 20 anos de idade. É uma fase que, apesar dos conflitos, tornase importante por marcar a passagem da proteção na infância, para a exposição na vida adulta ${ }^{1}$. É neste período complexo sob o ponto de vista social e psicológico, com mudanças nas várias áreas do indivíduo, além da física, que há uma grande busca por novas experiências, aliadas a comportamentos de impulsividade, ansiedade, insegurança, insatisfação e agressividade ${ }^{2}$.

O consumo de álcool e outras drogas por adolescentes têm crescido nos últimos anos, sendo considerado um grave problema de saúde pública, razão para estudos e envolvimento de diversos segmentos da sociedade na busca de soluções preventivas e de cuidado a esta população ${ }^{3}$. Os levantamentos epidemiológicos sobre o consumo de álcool e outras drogas entre os jovens no mundo e no Brasil mostram que é na passagem da infância para a adolescência que se inicia esse uso ${ }^{3-4}$. Geralmente, esse uso inicia-se pela experimentação de drogas lícitas, como o álcool e o tabaco em seus ambientes familiares, podendo haver uma transição para outros tipos de drogas, notadamente as ilícitas, como forma de aumentar o seu prazer, procurar outras emoções ou fugir de seus problemas ${ }^{4-6}$.

O uso de drogas na adolescência pode ser entendido como um comportamento ligado à experimentação própria dessa etapa da vida, às várias mudanças físicas e emocionais e à necessidade de pertencimento a um grupo de amizades, essencialmente ligado às várias operações psíquicas atuantes nesta fase ${ }^{7}$. Isso implica que, ao lado de toda a potência transformadora e questionadora própria desta etapa de vida, em que o sujeito adolescente busca sua afirmação pessoal perante seu meio social e cultural, o uso de drogas pode se tornar um comportamento mais frequente, podendo levar o adolescente a enfrentar problemas no desempenho de suas atividades nas várias esferas de sua vida, como escola, família, lazer, entre outros, relacionados ao uso de tais drogas. Esses problemas, também, podem ser mais frequentes quando esses indivíduos estão mais vulneráveis a fatores que podem representar maior risco para o uso e o abuso de drogas ${ }^{7}$.

Diante do exposto, o objetivo deste estudo foi avaliar a prevalência do uso e de problemas relacionados ao uso de álcool e outras drogas entre estudantes do $6^{\circ}$ ao $9^{\circ}$ ano do ensino fundamental de uma escola municipal de Uberaba - MG.

\section{MÉTODOS}

Trata-se de um estudo transversal de abordagem quantitativa. A abordagem quantitativa é caracterizada pelo emprego da quantificação, tanto nas modalidades de coleta de informações, quanto no tratamento delas por meio de técnicas estatísticas ${ }^{8}$. O estudo transversal é uma observação direta de determinada quantidade de indivíduos em uma única oportunidade, sendo que esta quantidade deve ser previamente planejada ${ }^{8}$.

A coleta de dados foi realizada em uma escola municipal de Uberaba, após autorização da secretaria municipal de educação e concordância da diretora da escola. Esta escola tinha, no período da coleta de dados, o maior número de alunos matriculados entre as escolas municipais, com cerca de 1600 alunos, recebendo crianças e adolescentes de diversos bairros da periferia do município, considerados em vulnerabilidade social. A coleta se deu em maio de 2013 e teve início mediante a assinatura do Termo de Consentimento Livre Esclarecido (TCLE) pelos pais ou responsáveis dos adolescentes participantes.

A amostra foi definida pelo critério de amostragem não probabilístico de casos típicos ${ }^{9}$, submetida à disposição de participação por parte de alunos de uma escola municipal do segundo ciclo do ensino fundamental, constituindo-se de 55 adolescentes. Foi selecionada uma escola municipal com maior número de alunos matriculados favorecendo maior captação de sujeitos. Nesta escola, foram selecionadas as classes do $6^{\circ}$ ao $9^{\circ}$ ano com maior número de alunos matriculados dos períodos manhã e tarde que recebem alunos com idade entre $10 \mathrm{a}$ 15 anos.

Os critérios de inclusão dos sujeitos para participação nesta pesquisa foram: ser aluno de escola municipal do ensino fundamental; ter idade entre 10 e 15 anos; estar matriculado nas séries compreendidas como $5^{\circ}$ ao $9^{\circ}$ ano e ter a autorização dos responsáveis legais, através da assinatura do TCLE. Os critérios de exclusão foram aqueles que não se aplicavam aos de inclusão; ou se o sujeito solicitasse sua retirada.

Os dados foram obtidos mediante a aplicação do instrumento DUSI - Drug Use Screening Inventory. O DUSI foi desenvolvido originalmente nos EUA em resposta a uma necessidade prática e objetiva de um questionário que avaliasse de forma rápida e eficiente os problemas associados ao uso de álcool e/ou drogas pelos 
adolescentes. No Brasil, ele foi adaptado e validado por pesquisadoras da Universidade Federal de São Paulo, para ser utilizado com a população de adolescentes ${ }^{10}$.

O DUSI é composto por 149 questões divididas em 10 áreas e de aplicação única, fornecendo um perfil da intensidade de problemas em relação ao (1) uso de substância; (2) comportamento; (3) saúde; (4) transtornos psiquiátricos; (5) sociabilidade; (6) sistema familiar; (7) escola; (8) trabalho; (9) relacionamento com amigos e (10) lazer/recreação. As questões são respondidas com "Sim" ou "Não", sendo que as respostas afirmativas equivalem à presença de problemas. Além das 10 áreas mencionadas, o DUSI possui uma "Escala da Mentira", composta por 10 questões, uma ao final de cada área, que foram acrescentadas com a finalidade de checar a existência de possíveis questionários inválidos.

O desenvolvimento deste estudo seguiu todas as normas éticas norteadoras dos trabalhos que envolvem seres humanos, sendo aprovado pelo Comitê de Ética em Pesquisa da Universidade Federal do Triângulo Mineiro UFTM, sob o número 2439/2013.

\section{RESULTADOS}

Foram convidados pela coordenação pedagógica da escola a participar da pesquisa um total de 144 alunos, sendo que destes apenas 59 tiveram o TCLE assinados por seus responsáveis e puderam responder o questionário. Dos 59 questionários respondidos, quatro deles foram excluídos, pois não respondiam o critério de inclusão de idade, constituindo-se a amostra do estudo em 55 adolescentes.

Os 55 questionários foram submetidos à análise estatística e ao teste de mentira proposto pelo DUSI. Para verificar eventuais diferenças de frequências dos resultados de acordo com o teste de mentira foi realizado o teste exato de Fisher. Este procedimento é necessário, pois pode indicar que eventuais diferenças significantes nas variáveis dependentes decorrem de reais diferenças entre os resultados. O teste foi empregado de maneira monocaudal e foram seguidas as especificações de Hollander e Wolfe ${ }^{11}$.

A partir do Teste de Mentira tem-se que 19 questionários $(34,55 \%)$, dos alunos mais novos, foram considerados inválidos. O Teste Exato de Fisher mostrou que os alunos mais velhos estão mais no grupo da verdade; que não há diferença em ser do sexo feminino ou do sexo masculino em relação ao resultado do teste de mentira, sendo considerados inválidos 33\% dos questionários das meninas e $36 \%$ dos questionários dos meninos.
Assim, foram analisados os questionários (DUSI) de 36 estudantes, sendo 22 do sexo feminino e 14 do sexo masculino, matriculados em uma escola municipal do $6^{\circ}$ ao $9^{\circ}$ ano do ensino fundamental da cidade de Uberaba, MG.

A análise do DUSI mostra em porcentagens, em quais áreas os adolescentes estão mais suscetíveis a terem problemas relacionados ao uso de álcool e outras drogas, distribuídos em densidades. A densidade absoluta do DUSI reflete a intensidade de problemas em cada área isoladamente, a densidade relativa reflete a contribuição percentual de cada área no total de problemas, e a densidade global que caracteriza a intensidade geral de problemas, que neste estudo foi de $32,7 \%$. A Tabela 1 mostra os resultados das densidades absoluta e relativa.

Tabela 1 - Média da Densidade Absoluta e Densidade Relativa de cada área do DUSI por sexo

\begin{tabular}{l|c|c}
\hline & $\begin{array}{c}\text { Densidade } \\
\text { absoluta \% }\end{array}$ & $\begin{array}{c}\text { Densidade } \\
\text { relativa \% }\end{array}$ \\
\hline Uso de substâncias & 34,9 & 10,5 \\
\hline Comportamento & 33,1 & 9,9 \\
\hline Saúde & 35,0 & 10,5 \\
\hline Transtornos psiquiátricos; & 30,3 & 9,0 \\
\hline Sociabilidade & 36,7 & 11,3 \\
\hline Sistema familiar & 43,7 & 13,1 \\
\hline Escola & 24,9 & 7,7 \\
\hline Trabalho & 3,6 & 1,0 \\
\hline $\begin{array}{l}\text { Relacionamento com } \\
\text { amigos }\end{array}$ & 35,7 & 10,8 \\
\hline Lazer/recreação & 52,5 & 16,2 \\
\hline
\end{tabular}

As áreas que apresentam maiores porcentagens de problemas referem-se à área do sistema familiar e à área de lazer/recreação. Na área do trabalho observa-se o menor valor. Da mesma forma, observa-se maior percentual na densidade relativa na área de lazer/recreação e menor percentual na área do trabalho.

Com relação à densidade global, a análise demonstrou que ser do sexo masculino ou do sexo feminino não influencia na intensidade geral de problemas, tendo-se média de $31,8 \%$ para meninas e $34,0 \%$ para os meninos.

Observa-se que os sujeitos de 10 anos apresentam um risco menor de problemas relacionados ao uso de álcool e outras drogas, comparados à média geral do grupo e em relação aos sujeitos mais velhos, embora entre os sujeitos, o grupo de 11 anos apresentou maior densidade global do que os sujeitos de 12 anos de idade. 
A Tabela 2 apresenta os resultados da média da densidade global por faixa etária dos participantes.

Tabela 2 - Média da densidade global por faixa etária dos participantes

\begin{tabular}{c|c}
\hline Idade & Densidade global \% \\
\hline 10 & 26,1 \\
\hline 11 & 34,0 \\
\hline 12 & 28,7 \\
\hline 13 & 33,9 \\
\hline 14 & 34,1 \\
\hline 15 & 34,2 \\
\hline
\end{tabular}

A análise das médias da densidade absoluta das dez áreas do DUSI em relação ao sexo dos sujeitos mostrou que meninos e meninas apresentam resultados semelhantes em quase todas as áreas. As áreas que mostraram maior diferença entre os sexos foram às áreas 'transtorno psiquiátrico' e 'sociabilidade'. O teste de Mann Whitney mostra que meninos têm maior prevalência de transtornos psiquiátricos quando fazem uso de drogas (área $04 \mathrm{p}=0,28$ ), e meninas maior prejuízo na sociabilidade (área $05 \mathrm{p}=0,038$ ).

Da mesma maneira, a análise das médias da densidade relativa das dezáreas do DUSI em relação ao sexo dos sujeitos mostrou semelhanças nos resultados de meninos e meninas, conforme apresenta a Tabela 3, obtendo-se menor densidade relativa na área do trabalho e maior densidade relativa na área do lazer/recreação. Tais resultados evidenciam que em todas as áreas o uso de drogas contribui de maneira semelhante na intensidade de problemas que esses adolescentes podem ter. Comparando-se esses resultados pelo sexo dos sujeitos, o teste de Mann Whitney mostra diferenças na intensidade dos problemas na área 5 , sendo maior para meninas $(\mathrm{p}=0,006) \mathrm{e}$ na área 08 maior para os meninos $(\mathrm{p}=0,40)$

Em relação à área do trabalho, uma hipótese para esse percentual poderia ser que, embora essa faixa etária ainda se encontrar fora do mercado formal de trabalho, talvez os meninos, em nossa cultura, tendam a preocuparem-se mais em serem produtivos. Quanto sociabilidade, o uso de drogas para este grupo de adolescentes parece influenciar mais meninas, que tendem a isolarem-se e afastarem-se do círculo de amigos.

Tabela 3 - Média do percentual das densidades absoluta e relativa por sexo

\begin{tabular}{c|c|c|c|c|c|c|c|c|c|c}
\hline Densidades/Sexo & Área 1 & Área 2 & Área 3 & Área 4 & Área 5 & Área 6 & Área 7 & Área 8 & Área 9 & Área 10 \\
\hline $\mathrm{daF}$ & 31,8 & 33,2 & 37,3 & 26,8 & 39,3 & 46,1 & 22,5 & 1,8 & $35 .$, & 48,9 \\
\hline $\mathrm{daM}$ & 39,8 & 32,9 & 31,4 & 35,7 & 32,7 & 39,8 & 28,6 & 6,4 & 35,7 & 58,3 \\
\hline $\mathrm{drF}$ & 9,7 & 10,0 & 11,3 & 8,2 & 12,3 & 14,2 & 7,3 & 0,5 & 11,1 & 15,3 \\
\hline $\mathrm{drM}$ & 11,6 & 9,9 & 9,2 & 10,3 & 9,6 & 11,4 & 8,3 & 1,8 & 10,2 & 17,7 \\
\hline
\end{tabular}

Legenda: $\mathrm{daF}=$ Densidade Absoluta do sexo feminino; daM = Densidade Absoluta do sexo masculino; $\mathrm{drF}$ = Densidade Relativa do sexo feminino; drM = Densidade Relativa do sexo masculino.

Os resultados apresentados na Tabela 4 evidenciam que os problemas devido ao uso de álcool e outras drogas (área 1) aumentam com a idade, sendo que o risco é nulo aos 10 anos, crescendo na adolescência e chegando a $57,1 \%$ aos 15 anos. Podemos verificar também que, na área 8 o risco de problemas no trabalho associados ao uso de drogas são baixos; e que os problemas no sistema familiar associados ao uso de drogas (área 6) estão presentes em todas as faixas etárias, crescendo conforme a idade aumenta.

Vale ressaltar que os resultados obtidos na área do lazer/recreação, com altos valores para todas as idades, revela grande risco de problemas associados ao uso de álcool e outras drogas.com o lazer/recreação desses adolescentes.

A análise da densidade relativa por área do DUSI mostrou que as áreas que mais contribuem no total de problemas relacionados ao uso de álcool e outras drogas são as áreas uso de substâncias, sistema familiar e lazer/ recreação, destacando-se o aumento de contribuição conforme o aumento de idade na área do uso de substâncias e o maior percentual na área do lazer/recreação, sendo maior a contribuição desta área do que o próprio uso de drogas. Destacam-se as baixas densidades nas áreas da escola e do trabalho, sendo esta última esperada tal resultado em virtude da idade da amostra. 
Ruzzi-Pereira A, et al. Problemas relacionados ao uso de álcool e outras drogas. Rev Ter Ocup Univ São Paulo. 2016 set./dez.;27(3):263-70.

Tabela 4 - Média da Densidade Absoluta de cada área do DUSI por idade

\begin{tabular}{c|c|c|c|c|c|c|c|c|c|c}
\hline Idade & Área 1 & Área 2 & Área 3 & Área 4 & Área 5 & Área 6 & Área 7 & Área 8 & Área 9 & Área 10 \\
\hline 10 & 0,0 & 41,0 & 32,0 & 33,0 & 25,7 & 30,0 & 23,0 & 2,0 & 24,3 & 41,7 \\
\hline 11 & 1,8 & 33,8 & 55,0 & 40,0 & 50,0 & 46,4 & 32,5 & 5,0 & 33,9 & 41,7 \\
\hline 12 & 35,7 & 25,0 & 25,0 & 22,5 & 17,9 & 53,6 & 30,0 & 0,0 & 28,6 & 50,0 \\
\hline 13 & 33,3 & 35,8 & 41,7 & 29,2 & 44,0 & 47,6 & 20,8 & 1,7 & 39,3 & 51,4 \\
\hline 14 & 50,4 & 31,3 & 31,9 & 29,7 & 36,6 & 42,0 & 25,9 & 3,8 & 38,8 & 56,8 \\
\hline 15 & 57,1 & 28,3 & 23,3 & 23,3 & 35,7 & 57,1 & 16,7 & 10,0 & 38,1 & 66,7 \\
\hline
\end{tabular}

Tabela 5 - Média da densidade relativa de cada área do DUSI por idade

\begin{tabular}{c|c|c|c|c|c|c|c|c|c|c}
\hline Idade & Área 1 & Área 2 & Área 3 & Área 4 & Área 5 & Área 6 & Área 7 & Área 8 & Área 9 & Área 10 \\
\hline 10 & 0,0 & 16,2 & 12,9 & 13,2 & 10,3 & 11,4 & 9,2 & 0,7 & 10,4 & 15,8 \\
\hline 11 & 0,6 & 10,4 & 16,5 & 11,2 & 14,5 & 13,5 & 9,5 & 1,3 & 10,1 & 12,5 \\
\hline 12 & 10,0 & 7,0 & 7,9 & 8,1 & 8,1 & 18,1 & 13,4 & 0,0 & 9,5 & 18,0 \\
\hline 13 & 10,4 & 9,6 & 12,0 & 7,8 & 12,9 & 13,7 & 5,6 & 0,6 & 11,2 & 16,1 \\
\hline 14 & 15,2 & 8,7 & 8,7 & 8,3 & 10,8 & 12,1 & 7,4 & 1,0 & 11,2 & 16,6 \\
\hline 15 & 16,0 & 7,9 & 6,5 & 6,4 & 10,1 & 16,2 & 4,6 & 2,8 & 10,6 & 18,9 \\
\hline
\end{tabular}

\section{DISCUSSÃO}

Nas dez áreas exploradas pelo instrumento aplicado aos sujeitos, os que mais chamaram a atenção pelo maior risco de problemas foram as relacionadas ao sistema familiar $(43,7 \%)$ e ao lazer/recreação $(52,5 \%)$.

Considerando esses resultados, pode-se perceber que há problemas na relação entre o adolescente e a família participantes da pesquisa, que resulta em risco ao uso de álcool e outras drogas.

A família é ainda um ambiente privilegiado para a melhoria da educação. Mesmo que o adolescente passe a viver mais em outros ambientes, como escolas, clubes e shoppings, é no meio da família que os valores morais e os padrões de conduta são adquiridos. Apenas quando esses valores morais não são adquiridos da melhor forma durante a infância, é que os outros ambientes influenciarão na adolescência ${ }^{12}$.

Estudos mostram que filhos cujos pais estão mais atentos às atividades por eles desenvolvidas apresentam menor envolvimento com álcool e outras drogas ${ }^{13}$. As relações familiares saudáveis desde o nascimento da criança servem como fator de proteção para toda a vida e, de forma muito particular, para o adolescente. No entanto, problemas enfrentados na adolescência, plantados na infância, têm um contexto de realização muito mais ampliado $^{13-16}$.
A área relacionada ao lazer/recreação representa a de maior risco de problemas relacionados ao uso de álcool e outras drogas. O lazer é considerado como algo relevante para a vida das pessoas, estando diretamente ligado a uma questão de saúde. O lazer envolve um conjunto de atividades que o indivíduo realiza com prazer visando à satisfação pessoal, o descanso, o divertimento, a participação social voluntária, expressando, assim, a cultura de um grupo ou sociedade ${ }^{14}$.

Oferecer alternativas de lazer aos adolescentes é uma estratégia relevante de prevenção do uso de drogas, uma vez que as atividades físicas e a participação em atividades extracurriculares são consideradas como recursos importantes neste contexto. Nesta mesma linha, modos atrativos de ocupar o tempo livre podem funcionar como fatores de proteção na adolescência ${ }^{15}$. Além disso, o investimento em atividades de cultura e esporte poderiam influenciar no desenvolvimento físico e emocional dos adolescentes, numa melhoria do desempenho escolar, e até no afastamento dessas pessoas do consumo de drogas e de situações que as deixam vulneráveis à violência ${ }^{16}$.

Embora existam alguns estudos sobre fatores de risco para uso de drogas entre adolescentes no Brasil, a compreensão sobre tais fatores ainda é controversa. Além de fatores sociodemográficos como sexo, idade e classe social, os estudos indicam associação do uso de drogas com envolvimento parental ou familiar no consumo de 
álcool ou drogas, não criação por ambos os pais, baixa percepção de apoio paterno e materno, amigos que usam drogas, ausência de prática religiosa, bem como menor frequência à prática de esportes ${ }^{16-19}$.

As menores densidades obtidas foram nas áreas "escola" (24,95\%) e "trabalho" (3,6\%). A escola pode ser considerada como agente transformador, é um espaço privilegiado de encontros e interações entre jovens. Ainda, por juntar em seu interior a comunidade de pares e por ter fortes instrumentos de promoção da autoestima e do autodesenvolvimento em suas mãos, o ambiente escolar pode ser um fator fundamental na potencialização de resiliência dos adolescentes ${ }^{10}$. No entanto, mesmo no âmbito educacional, existem fatores específicos que predispõem os adolescentes ao uso de drogas, como por exemplo, a falta de motivação para os estudos e o mau desempenho escolar.

O trabalho não está diretamente relacionado com a faixa etária desta população, porém observa-se alguma preocupação com os adolescentes de 14 e 15 anos. Nessa idade, os adolescentes já podem entrar no mercado formal de trabalho como aprendizes.

O Estatuto da Criança e do Adolescente (ECA) estabelece diretrizes para a proteção da atividade laboral na adolescência. É proibido o trabalho a menores de 14 anos, exceto na condição de aprendiz ${ }^{20}$.

Por sua vez, a contratação de aprendizes, regulamentada pelo Decreto $\mathrm{n}^{\mathrm{o}} 5598 / 2005$, assegura formação técnico-profissional e realização de atividades compatíveis com a vida escolar e com o desenvolvimento físico, psicológico e moral do aprendiz ${ }^{21}$. Assim, o Decreto impõe limites ao trabalho dos adolescentes, visando proporcionar reais condições de aprendizagem e minimizar os impactos do trabalho precoce.

É possível pensar que os alunos que apresentem maior risco de problemas relacionados ao uso de álcool e outras drogas não consigam frequentar a escola de maneira adequada, tampouco desempenhar trabalho de aprendiz. Assim, a escola que estimula a participação social e o engajamento em atividades produtivas poderia atuar como um fator de proteção ao uso de drogas.

No presente estudo não houve diferenças entre ser do sexo masculino e do sexo feminino em relação à intensidade geral de problemas relacionado ao risco do uso de álcool e outras drogas. Embora haja ainda poucos estudos com meninas, tal resultado concorda com que se vem demonstrando sobre o comportamento feminino em relação ao uso de substâncias lícitas e ilícitas, que se tornou bastante semelhante ao dos meninos nos últimos anos, principalmente na adolescência. Assim, existe uma tendência em adotarem os mesmos comportamentos, buscando a aceitação pelo próprio grupo ${ }^{9}$.

As maiores diferenças entre os resultados para o sexo masculino foram na área relacionada aos transtornos psiquiátricos e na área relacionada ao trabalho.

Em relação aos transtornos psiquiátricos, o transtorno de conduta é o mais comumente relacionado aos meninos, associado ao uso de álcool e outras drogas. É também um transtorno frequente em adolescentes mais velhos, independente do sexo. Os transtornos de conduta abrangem os comportamentos de risco que podem prejudicar a saúde física e mental do adolescente. São comportamentos repetitivos e persistentes, em que o indivíduo viola direitos básicos dos outros, normas ou regras sociais, iniciando comportamentos agressivos e reagindo agressivamente a outras pessoas ${ }^{22}$.

No presente estudo a amostra apresenta o consumo de álcool e outras drogas a partir dos 13 anos, aumentando o risco conforme a idade aumenta. Tal resultado concorda com a pesquisa de $\mathrm{Strauch}^{23}$, que observou a prevalência do consumo de álcool e outras drogas com relação ao aumento da faixa etária, a partir dos 13 anos.

A pesquisa de Strauch $^{23}$ também mostrou que crianças com 11 anos de idade já haviam experimentado bebidas alcoólicas, percebendo o uso cada vez mais precoce, diferente dos achados nesta amostra, em que as crianças de 10 a 12 anos pouco apresentaram esse comportamento.

Outro estudo também aponta que com o aumento da escolaridade, a probabilidade aumenta em até três vezes mais para o uso de drogas, pois, se por um lado há a formação educativa, por outro os adolescentes podem estar se desvinculando dos familiares, associando-se a grupos de amigos, condições favoráveis para o envolvimento com fatores de risco como experimentar drogas ${ }^{24}$.

\section{CONCLUSÃO}

O presente estudo possibilitou avaliar o risco do uso de álcool e outras drogas entre alunos do $6^{\circ}$ ao $9^{\circ}$ ano de uma escola municipal de Uberaba do segundo ciclo do ensino fundamental e, com isso, contribui na compreensão sobre proteção e cuidado de adolescentes em relação ao uso de drogas. Conclui-se que o risco de problemas relacionados ao uso é igual para meninas e meninos; e que o início do uso e dos problemas a ele relacionados se dá a partir dos 13 anos.

Ressalta-se a necessidade de investimentos em lazer/recreação saudáveis e adequados aos interesses dessa população, visto ser esta a área de maior risco para 
o uso de álcool e outras drogas e para os problemas a ele relacionados.

Outros estudos relativos ao lazer e à recreação de estudantes do ensino fundamental e sua relação de risco e proteção ao uso de drogas devem ser realizados, para verificar se o mesmo ocorre em diferentes populações na mesma porcentagem, reconhecendo-se este elemento como um limite deste estudo.

Destaca-se, ainda, o uso de drogas ser um fenômeno multicausal e, para que se possa destinar ações eficientes de prevenção e atenção ao usuário, se faz necessário o envolvimento de profissionais de diversas áreas para elaborar estratégias e propostas de intervenção, inclusive a inserção destes profissionais no ambiente escolar, trabalhando juntamente com os professores na elaboração de atividades de educação em saúde, direcionadas ao adolescente e sua família; e no planejamento de políticas voltadas à construção de ambientes protetores e saudáveis para melhorar a qualidade de vida dos alunos e da comunidade.

No Brasil, o Ministério da Educação e Cultura, por meio das Secretarias de Educação Básica e de Educação
Continuada, Alfabetização, Diversidade e Inclusão em parceria com as Secretarias Municipais e Estaduais de Educação, vem implementando programas nas escolas com vistas a capacitar profissionais de educação, membros dos conselhos de educação, conselhos escolares, além de profissionais da saúde, assistência social, conselheiros tutelares, entre outros profissionais ligados à Rede a fim de: garantir os direitos da população escolar e o enfrentamento e prevenção das violências no contexto escolar, como no caso do Programa Educação que Protege; proporcionar melhoria da qualidade de vida da população brasileira, pelo Programa Saúde na Escola; e capacitar a comunidade escolar para o manejo de situações relacionadas ao uso de substâncias pelos escolares, de forma preventiva e não excludente, pelo curso Prevenção do Uso de Drogas para Educadores da Educação Básica. Além dos objetivos supracitados, tais Programas e ações podem aproximar o estudante do ambiente escolar, compondo uma comunidade escolar, no sentido em que recebem atenção adequada e fortalecem os vínculos com os profissionais que estão sendo melhor preparados para o enfrentamento das situações adversas.

\section{REFERÊNCIAS}

1. Coordenação de Desenvolvimento de Programas e Políticas de Saúde. Manual de atenção à saúde do adolescente. São Paulo: SMS; 2006 [citado 2 jul. 2013]. Disponível em: http://www.tele.medicina.ufg.br/files/palestrasmaterial/ Manual_do_Adolescente.pdf.

2. Almeida JF, Carvalho KD, Cruz STM, Carvalho MFAA, Figueiredo RGT. Uso de álcool entre estudantes de escolas da rede pública de ensino. Rev Enferm UFPE. 2013;7(2):397406. doi: 10.5205/reuol.3073-24791-1-LE.0702201310.

3. Carlini ELA, Noto AR, Sanches ZM, Carlini CMA, Locatelli DP, Abeid LR, et al. VI levantamento nacional sobre o consumo de drogas psicotrópicas entre estudantes do ensino fundamental e médio das redes pública e privada de ensino nas 27 capitais brasileiras: 2010. São Paulo: CEBRID/UNIFESP; 2010.

4. Marti NC, Stice E, Springer DW. Substance use and abuse trajectories across adolescence: A latent trajectory analysis of a community-recruited sample of girls. J Adolesc. 2010, 33(3):449-61. doi: 10.1016/j.adolescence.2009.06.005.

5. Barreto SM, Giatti L, Casado L, Moura L, Crespo C, Malta DC. Exposição ao tabagismo entre escolares no Brasil.

Ciênc Saude Coletiva. 2010;15(supl. 2):3027-34. doi: 10.1590/S1413-81232010000800007.

6. Stone AL, et al. Review of risk and protective factors of substance use and problem use in emerging adulthood. Addict Behav. 2012;37(7):747-75. doi: 10.1016/j. addbeh.2012.02.014.

7. Raupp L, Sapiro CM. Adolescência, drogadição e políticas públicas: recortes no contemporâneo. Estud Psicol. 2009;26(4):445-54. doi: 10.1590/S0103166X2009000400005.

8. Klin $\mathrm{CH}$, Bloch KV. Estudos seccionais. In: Medronho RA, Bloch KV, Luiz RR, Werneck GL. Epidemiologia. São Paulo: Atheneu; 2009. p.193.

9. Sampieri RH, Collado CF, Lucio PB. Seleção da amostra. In: Sampieri R H, Collado CF, Lucio PB. Metodologia de pesquisa. 3a ed. São Paulo: McGraw-Hill; 2006. p.248-83.

10. Micheli D, Sartes LMA. A detecção do uso abusivo em adolescentes usando o DUSI e o T-ASI. In: Curso Sistema para detecção do uso abusivo e dependência de substâncias psicoativas: encaminhamento, intervenção breve, reinserção 
social e acompanhamento. Brasília: Secretaria Nacional de Políticas sobre Drogas; 2008.

11. Hollander M, Wolfe DA. Nonparametric statistical methods. 2nd ed. New York: John Wiley \& Sons; 1999.

12. Galduróz JCF, Sanchez ZM, Opaleye ES, Noto AR, Fonseca AM, Gomes PLS, et al. Fatores associados ao uso pesado de álcool entre estudantes das capitais brasileiras. Rev Saúde Pública. 2010;44(2):267-73. doi: 10.1590/S003489102010000200006.

13. Vieira PC, Aerts DRGC, Freddo SL, Bittencourt A, Monteiro L. Uso de álcool, tabaco e outras drogas por adolescentes escolares em um município do Sul do Brasil. Cad Saúde Pública. 2008;24(11):2487-98. doi: 10.1590/ S0102-311X2008001100004.

14. Schenker M, Minayo MCS. Fatores de risco e de proteção para o uso de drogas na adolescência. Ciênc Saúde Coletiva. 2005;10(3):707-17. doi: 10.1590/S141381232005000300027.

15. Guimarães ABP, Hochgraf PB, Brasiliano S, Ingberman YK. Aspectos familiares de meninas adolescentes dependentes de álcool e drogas. Rev Psiq Clin. 2009;36(2):69-74. doi: 10.1590/S0101-60832009000200005.

16. Paiva FS, Rozani TM. Estilos parentais e consumo de drogas entre adolescentes: revisão sistemática. Psicol Estud. 2009;14(1):177-83. doi: 10.1590/S141373722009000100021.

17. Pratta EMM, Santos MA. Lazer e uso de substâncias psicoativas na adolescência: possíveis relações. Psicol Teor Pesqui. 2007;23(1):43-52. doi: 10.1590/S010237722007000100006

Recebido em: 15.1 .15

Aceito em: 05.09.16
18. Monteiro EMLM, Neto WB, Gomes IMB, Freitas RBN, Brady CL, Moraes MUB. Violência contra criança e adolescente: rompendo o silêncio. Rev Rene. 2009;10(3):107-16.

19. Cavalcante MBPT,Alves MDS, Barroso MGT.Adolescência, álcool e drogas: uma revisão na perspectiva da promoção da saúde. Esc Anna Nery Rev Enferm. 2008;12(3):555-59. doi: 10.1590/s1414-81452008000300024

20. Brasil. Lei $n^{\circ} 8.069$, de 13 de julho de 1990. Dispõe sobre o Estatuto da Criança e do Adolescente e dá outras providências. D.O.U.; 16 jul. Brasília, DF; 1990 [citado 2013 jun 12]. Disponível em: http://www.planalto.gov.br/ ccivil_03/leis/18069.htm.

21. Brasil. Decreto $\mathrm{n}^{\circ}$ 5598, de $1^{\text {o }}$ de dezembro de 2005. Regulamenta a contratação de aprendizes e dá outras providências. D.O.U.; 2 dez. Brasília, DF; 2005 [citado 2013 jul 5]. Disponível em: http://www.planalto.gov.br/ ccivil_03/_ato2004-2006/2005/decreto/d5598.htm.

22. Cruzeiro ALS, Silva RA, Horta BL, Souza LDM, Faria $\mathrm{AD}$, Pinheiro RT, et al. Prevalência e fatores associados ao transtorno da conduta entre adolescentes: um estudo de base populacional. Cad Saúde Pública. 2008;24(9):2013-20. doi: 10.1590/S0102-311X2008000900007

23. Strauch ES, Pinheiro RT, Silva RA, Horta BL. Uso de álcool por adolescentes: estudo de base populacional. Rev Saúde Pública. 2009;43(4):647-55. doi: 10.1590/S003489102009005000044.

24. Jinez LJ, Souza JRM, Pillon SC. Uso de drogas e fatores de risco entre estudantes de ensino médio. Rev Latino-Am Enfermagem. 2009;17(2):246-52. doi: 10.1590/S010411692009000200017. 